\title{
Research on Pedestrian Red Light Running Violation Based on the Social Conformity
}

\author{
Zan Hao \\ College of Economics and Management \\ Hebei University of Science and Technology \\ Shijiazhuang, China \\ 1027571874@qq.com
}

\author{
Yan Gao \\ College of Economics and Management \\ Hebei University of Science and Technology \\ Shijiazhuang, China \\ 26761983@qq.com
}

\begin{abstract}
Pedestrian Red Light Running Violation that Chinese-style crossing the road is a general conformity behavior, the phenomenon of running red lights collectively and not obeying the traffic rules are general, seriously affecting the traffic order and spiritual civilization construction. This paper analyzed the yield of conformity by using complete information static game, and studied the reason of Chinese-style crossing. In the final we put forward a problem-solving measure that introducing a corresponding standard mechanism. For Chinese-style crossing the road, basing on the analysis of static game of complete information, in order to solve the problem that running the red light, we can make an effort from two aspects. The one is to strengthen law enforcement of traffic policemen. The other one is to severely punish those who run the red light. Only by these ways can we solve the problem of Chinese-style crossing the road.
\end{abstract}

Keywords- Spiritual civilization construction; Pedestrian Red Light Running Violation; Complete information; Static game; Social Conformity

\section{INTRODUCTION}

The problem of pedestrian red light running violation is very prominent, and the phenomenon of not obeying the traffic rules and running red lights are both general ${ }^{[1-2]}$. A province done a survey for 13 prefecture-level cities, finding that the violation rate of crossing the road is $44.16 \%$, and in terms of the school students, $83 \%$ respondents acknowledged that he often crosses the road ${ }^{[3]}$.

For pedestrian red light running violation, the solving methods still remain at strengthening propaganda to make the masses get a education of socialist concept of honor and disgrace. People can get such an education that stopping at a red light and going at a green light from they enter the kindergartens. "No forbidding an entry by the red light, where is the pass through by the green light", this principle is still remain at propaganda, aiming at improving civil competence, and by contrast the punitive measures for crossing the road are seldom. Currently, Wenzhou city start to punitive the illegal behavior of running the red light and jaywalking, and unify the punishment intensity that imposing a fine of 5 yuan, however, the phenomenon of crossing the road still exist through several bans. Some people think that taking the illegal status of China intersection pedestrian red light running phenomenon as an example, and running from the perspective of behavioral science to analyze the main causes of pedestrian red light the causes of bus delay are analyzed ${ }^{[4][5]}$.Some people think that the question is why the red-light running phenomenon widely exists. After a study on the basic principles of jurisprudence and the theories on economics and sociology, conclusion can be drawn that such existence has its rationality considering the various aspects about the violators, the law environment and the social legal system ${ }^{[6]}$. In view of the current situation, most of the analysis of the pedestrian red light running violation, stays on the qualitative analysis. They just went up from image analysis and put forward the countermeasure and suggestion. They don't have a deeper analysis. This article from the perspective of economic man.

So aiming at the phenomenon of pedestrian red light running violation, this paper made a cost-benefit analysis from the view of game theory to find the reason and proposed a concrete solving method ${ }^{[7]}$.

\section{PROBLEM DESCRIPTION AND MODEL ASSUMPTION}

Problem description: The both sides in the game that running the red light are traffic policemen and pedestrian groups. The action of the both sides has no sequence, that is the both sides are gaming at the same time. The traffic policemen and the pedestrian groups both have two strategies, the strategies of traffic policemen are supervision and no supervision, and the strategies of pedestrian groups are running the red light and not running the road. They can take a strategy independently. As a rational broker, the choice is made basing on the consideration of cost-benefit inevitably.

Model assumption: (1) Supposing the sides are both the limited rational agent and risk-neutral. (2) Suppose the social system is relatively perfect and the social spiritual civilization needs to be improved.

\section{ESTABLISHMENT AND ANALYSIS OF THE MODEL}

\section{A. The existence of Nash equilibrium}

When there is Nash equilibrium between both sides, if supposing the both sides have a same revenue structure, we can express the game combination by payoff matrix ${ }^{[7]}$. We show it in Table 1. 
Table 1

\begin{tabular}{|c|c|c|}
\hline & No supervision & Supervision \\
\hline Not running the road & Isc, Ifc & Isc*, Ifu \\
\hline Running the road & Isu, Ifc* & Isu $^{*}$, Ifu* \\
\hline
\end{tabular}

In Table 1, Isc, Ifc denote the benefit that not running the red light and no supervision respectively; Isu*, Ifu*denotes the benefit that running the red light and supervision respectively; Isc*, Ifu denotes the benefit that not running the red light and supervision; Isu, Ifc* denotes the benefit that running the red light and no supervision respectively. If we suppose both sides in the game are rational, then when the benefit values are different, the equilibrium results of the model are also different.

- (A) When Isc $>$ Isu, Isc*>Isu*, Ifc $>$ Ifu, Ifc*>Ifu*, the choice of both sides are not running the red light and no supervision respectively. This is the only Nash equilibrium status and a very ideal status. It illustrates that citizen's moral level is in the continuous improvement and it have a benefit to constructing socialist harmonious society.

- (B) When Isc $<$ Isu, Isc*<Isu*, Ifc $<$ Ifu, Ifc*<Ifu*, the choice of both sides are running the red light and supervision respectively, and this is the only Nash equilibrium. This status is an effective measure for solving the current social conformity behavior.

- (C) When Isc $>$ Isu, Ifc $>$ Ifu, Isu<Isu*, Ifc*<Ifu*, the choice of both sides are not running the red light and no supervision respectively and choice of running the red light and supervision are both Nash equilibrium. The two states both have a benefit to the progress and development of social civilization.

- (D) When Isc $>$ Isu, Ifc $<$ Ifu, Isu $<$ Isu*, Ifc* $>$ Ifu*, the choice of not running the red light and supervision and the choice of running the red light and no supervision are both Nash equilibrium. The choice of not running the red light and supervision is equal to wasting the social resource, and this more ideal status is difficult to achieve in the realistic society. The choice of running the red light and no supervision is general in the realistic society, the reason is that the cost of running the red light is too low and the punishment when there is no supervision is also low, nearly zero.

Therefore, when there is only one game, each participant focus only on short-term benefit. For the game between pedestrian groups and traffic polices, if the punishment for the betrayer is too small or unreliable, then the possibility of cooperation about both sides in the game will be small. In order to realize and maintain the choice that not crossing the road and no supervision, it needs a relatively independent repeated game and the trust between the both sides. That is to say, if the game repeats many times, then the participators will sacrify immediate benefits for seeking long term benefits and choose different equilibrium strategies. The result is that the cooperation impossibly realized in one-time game becomes possible, achieving a more efficient equilibrium. From the analyze above, we can draw a conclusion that traffic polices will not being any punished, and the punishment for pedestrian groups in the reason of running the red light also will be small. Therefore, the solving method is to severely punish those who run the road, and to strengthen supervision and punishment intensity for omission of traffic polices.

\section{B. Not existence of Nash equilibrium}

When there is no Nash equilibrium between both sides, we can use the game between thief and guard to analyze the Chinese-style crossing the road phenomenon.

Table 2

\begin{tabular}{|c|c|c|}
\hline & No supervision & supervision \\
\hline Running the road & $\mathrm{V}, \mathrm{D}$ & $-\mathrm{P}, 0$ \\
\hline Not running the road & $0, \mathrm{~S}$ & 0,0 \\
\hline
\end{tabular}

From Table 2 we can see that pedestrian groups have two choice of running the red light and not running the road, and traffic polices can choose no supervision, strict supervision and laxative supervision. If traffic polices choose no supervision, then the behavior of running the red light of pedestrian groups must be happened. Now the benefit of pedestrian groups is $\mathrm{V}$, and the benefit of traffic polices is -D; If pedestrian groups choose running the red light and traffic polices choose supervision, then people who run the red light will be punished and gain a loss -P. If traffic polices choose no supervision and pedestrian groups not cross the road, then social supervision person gain a benefit S. From Table 2 we can see obviously that social supervision person and the conformity behavior participators both have no optimal strategy, and there is a connection between running the red light or not running the red light and supervision or no supervision. Suppose the probability of running the red light is $\mathrm{Pt}$, and the probability of supervision is $\mathrm{Pg}^{*}$, and we analyze the mixed strategy of pedestrian groups basing it. Here $\mathrm{Pt}^{*}$ denotes the optimum probability level of running the red light by pedestrian groups, and the optimum probability level that not running the red light is $1-\mathrm{Pt}^{*}$. Then we can compute the expected revenue of no supervision is $\mathrm{S}(1$ $\mathrm{Pt})+(-\mathrm{D}) \mathrm{Pt}$ it is shown in Figure 1 . 


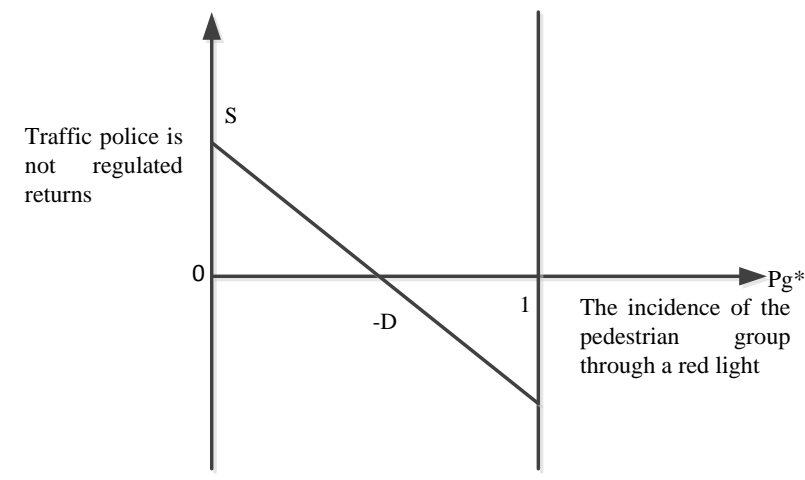

Figure 1

When the probability of running the red light by pedestrian groups exceeds $\mathrm{Pt}^{*}$, then the benefit of traffic policemen is less than zero. Therefore traffic policemen will choose supervision, and it is not desirable for people who participate running the road. On the other hand, if the probability of running the red light is less than $\mathrm{Pt}^{*}$, then the expected benefit of traffic policemen will be more than zero, so traffic policemen choosing no supervision is remunerative. Therefore, though the probability of running the red light raises a bit, if and only if it no more than $\mathrm{Pt}^{*}$, traffic policemen will choose no supervision. When the participators are not afraid to be punished, then the benefit of pedestrian groups will be more and more, and the behavior of running the red light will be tending to $\mathrm{Pt}^{*}$. The equilibrium is that the probability of participators choosing running the red light is $\mathrm{Pt}^{*}$, and the probability of choosing not running the red light is 1-Pt*. But in fact, in order to let the participators have no opportunity, traffic policemen must choose a mixed strategy in a specific probability distribution. In the same way, the best probability choice of traffic policemen is $\mathrm{Pg}^{*}$ and $1-\mathrm{Pg}^{*}$, it is shown in Figure 2.

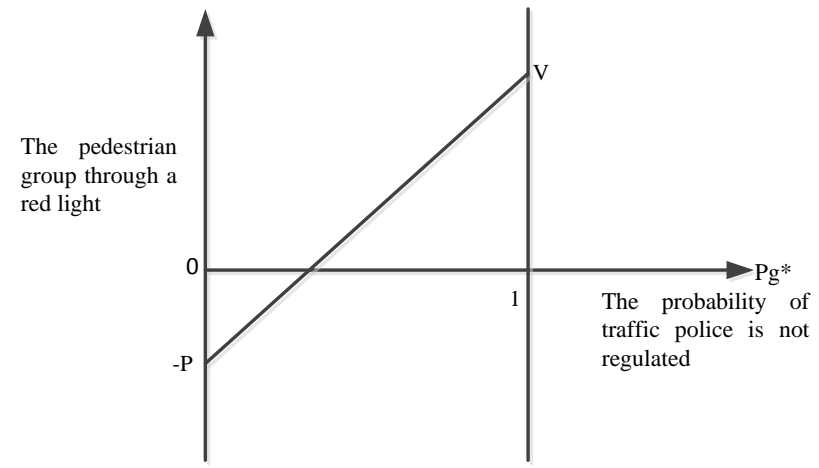

Figure 2

In my opinion, the probability of running the red light is not only associated with the punishment intensity for this behavior, but also associated with the punishment intensity for the triffic policemen.

\section{COUNTERMEASURES AND SUGGESTIONS}

For the problem of pedestrian red light running violation, whether there is Nash equilibrium or not, we should solve it from two aspects. The one aspect is from the view of persons who supervise in the society. The chief persons who supervise the social traffic are traffic policemen. Traffic policemen should serve people for the reason that they have a authority conferred by people, and should be responsible for a due supervision duty to maintain social fairness. If traffic policemen do not carry out his responsibility carefully, we can introduce the third party supervision to supervise traffic policemen. The specific practice is introducing social groups to supervise traffic policemen for their law enforcement effect. Strengthen the supervision of media in the same time, and try to be fair. Analyze from the other part, people who participate the behavior of running the red light are all treat the problem from the view of broker, which is taking economic benefits as the starting point, and will do anything as long as they have good prospects of gain. Therefore, there is a need to severely punish those who run the road. Specific practices are list in the following:

- (1) Raising the penalty for people who participate the behavior of running the road ${ }^{[8]}$. For the practice that Wenzhou city unify the punishment by 5 yuan for the behavior of running the red light and Jaywalking, in my opinion, it is a little less. Penalty in American changes from 50 dollars to 500 dollars, and it is different among different areas. There is a need to restudy traffic regulations in the same time. To sum up, we should raise the penalty, for example, punish 100 yuan one time.

- (2) For parties who do not accept the fine, we will let them participate in the traffic persuasive management in the base of voluntary principle for three hours, and we will prepare uniforms in advance. For parties who not only do not accept the fine, but also do not want to be a traffic persuade member, traffic policeman should record their ID information, and feed it back them to their companies. 
- (3) Establishing personal trustworthiness file and citizen moral files ${ }^{[9][10]}$. For people who not only do not accept the fine, but also do not want to be a traffic persuade member, traffic policemen should record the personal credit and feed it back to related parties ${ }^{[11]}$. Owing to the low reputation degree, people who running the red light cannot lend long-term loans like other people. As to shortterm loans, the bank will regulate a higher interest rates than other people.

\section{CONCLUSIONS}

For pedestrian red light running violation, basing on the analysis of static game of complete information, in order to solve the problem that running the red light, we can make an effort from two aspects. Establishing personal trustworthiness file and citizen moral file. The one is to strengthen law enforcement of traffic policemen. The other one is to severely punish those who run the red light. For parties who do not accept the fine, we will let them participate in the traffic persuasive management in the base of voluntary principle for three hours, and we will prepare uniforms in advance. Raising the penalty for people who participate in the behavior of running the road. The pedestrian red -light not only affect the city, but also a threat to its own security. In order to solve the problem, not only needs the government's need to play a role in guidance, but also they should take measures to control the things happen. People should set up correct concept of transportation, and improve their own quality. For people who not only do not accept the fine, but also do not want to be a traffic persuade member, traffic policemen should record the personal credit and feed it back to related parties. Owing to the low reputation degree, people who running the red light cannot lend long-term loans like other people. As to short-term loans, the bank will regulate a high interest rates than other people. Only by these ways can we solve the problem of Chinese-style crossing the road.

\section{REFERENCES}

[1] Shi jin Sun ,"Social Psychology”,Fu dan university press,2003.

[2] Chen ming liang and Gao chun yu "Software Download at the Consumer Bandwagon Effect",JOURNAL OF ZHE JIANG UNIVERSITY.2013-03-10.

[3] Jian sheng Song, "Red light why Hard to Ban," JOURNAL OF SAFETY AND HEALTH,2006.

[4] Si rong Chen ,"Complete information static game analysis of supplier and the manufacturer",JOURNAL OF SCIENCE AND TECHNOLOGY INFORMATION.2008-06-10.

[5] Gui yin Cao,"Behavior Analysis and Solutions for Pedestrian Red Light Running Violation”,JOURNAL OF Beng Bu College.201304-20.

[6] Tang yun, " Study on Red-light Running Problems of Pedestrians",JOURNAL OF He Nan Police Academy.2012-02-05.

[7] Shi yu Xie, "Economic Game Theory",Fu dan university press,2006.

[8] HouYu , Chen Haiyu , "Optimal Sewage Charges of A Threestage Game for the Government, Polluting Firms and the Environment Firms ",JOURNAL OF NANKAI ECONOMIC STUDIES.2013-02-22.

[9] Cong man wang,Chang li WU," Research on the Human Resource Situation and Countermeasures of Shijia zhuang National Biological Industry Base " ,JOURNAL OF HE BEI ACADEMIC.2012-01-01.
[10] Cong man wang,Xue Bai,Yu Zhang, " Basic Elements and Relations of in the Culture of a University Class",JOURNAL OF HEBEI NORMAL UNIVERSITI.2013-08-15.

[11] Yan Wang, "A Dynamic Game Analysis on Emergency Management of Construction Accident",International Conference on Engineering and Business Management,2011-03-22. 